\title{
CLIMATE ANALYSIS IN IOWA USING XML AND SPATIOTEMPORAL DATASET-NC94
}

\author{
Sugam Sharma ${ }^{1}$, Shashi Gadia ${ }^{1}$, Niranjan Kumar ${ }^{2}$, \\ Valliappan Narayanan ${ }^{3}$ and Xinyuan Zhao ${ }^{1}$ \\ ${ }^{1}$ Department of Computer Science, Iowa State University, Ames, Iowa, USA \\ ${ }^{2}$ Cerner Corporation, Kansas City, Missouri, USA \\ ${ }^{3}$ Intel Corporation, Beaverton, Oregon, USA \\ sugam.k.sharma@gmail.com
}

\begin{abstract}
NC94 is a spatiotemporal (agricultural) dataset of North Central Region in USA and vastly used in various research domains. In this research work we exploit XML on NC94 dataset to assess the impact of extreme temperatures in Iowa. The variation in climate is one of the factors that affect human life. The results of this research help to identify the counties with suitable modest climate with least variations.
\end{abstract}

\section{KEYWORDS}

NC94, XML, Coldness, Hotness, Iowa

\section{INTRODUCTION}

Spatiotemporal databases have potential usage in diversified research domains and applications such as geography, agriculture, city planning, aerospace research, environmental study, traffic navigation and etc. In this work spatiotemporal agriculture dataset has been exploited. For last 50 year, The North Central Regional Association of Agricultural Experiment Station (NCRA) [11] in the United States is collecting and validating agricultural databases. The association has collected an important, internally consistent dataset called the NC94 which is intensively used to facilitate crop and risk analysis, pest management and forecasting, and available to public download through the internet. It is a compilation of 30 years county-level crop-climate-soil database, consisting of temperature, precipitation, and, crop yield and soils data. It is considered as an important resource in the agricultural community and careful analysis of the dataset from different faces would develop significant understanding for scientists, public, farmers, planners, and policy makers to improve crop production, and policy development. In this research, only the climatic data of NC94 dataset has been used and is loaded in a local storage called CanStoreX before any executable query is applied.

CanStoreX is an XML storage technology known as a Canonical Storage for XML [2]. It paginates a large XML document and stores it in the storage. Thus CanStoreX is used as a backend for storing NC94 data hiding the heterogeneity in climate, crop, and soil data in order to allow the user a simple view of counties as objects where geographical and time dimensions are implicit.

The execution of ParaSQL queries on NC94 dataset stream out the desired data which can either be seen on DOS console or can be stored into a text file and can be used as input for further processing. The streamed out dataset consists of many attributes and few of them are of our great interest and in this paper we consider the attributes of climate. 
The climate in Iowa significantly varies over the year due to its latitude and interior continental location. A summer maximum rainfall during almost half of the year (warmer months) can be seen due to the moist southerly flow from the Gulf of Mexico. A relatively dry but cold weather can be observed in winter session. The cause of this is the northwesterly flow of dry Canadian air. The Pacific Ocean also is partially responsible for climate variation in Iowa throughout the year. Due to air masses drift across the western United States and Iowa the weather becomes mild and dry comparatively. During the summer the hot and dry winds emerging from the Southwest Desert flow into Iowa and that causes unusual high temperatures.

The motivation of this research is to analyze the climatic conditions in all counties of Iowa using XML and NC94 dataset. A county having suitable modest climate (least climatic variation in terms of hotness, coldness) over the 30 years period can be recommended for various purposes, for example; to analyze the climate to choose a site suitable to establish smart homes for senior citizens where they can enjoy comfortable and independent lifestyle. As it is already mentioned that NC94 dataset is a collection of 30 years period so it can be believed, the use of this data is good enough to produce more accurate prediction about climatic patterns. Climate in a county is considered cold if the average temperature in that county over 30 years period (1971-2000) is less than $0^{\circ} \mathrm{C}$ and degree of coldness among counties varies depending upon the average temperature below $0^{\circ} \mathrm{C}$ and a county whose average temperature for the same duration is greater than $20^{\circ} \mathrm{C}$ is considered as the hot county and degree of hotness among counties varies too. We confine this research to Iowa only and in visualization to preserves the integrity of the map of Iowa, we do not vanish those counties, which are not qualifying the criterion rather paint with the most diluted shade of the base color. To demonstrate the coldness and hotness in a county blue and red are used as the base colors. The variations in the degree of coldness and hotness are distinguished by varying the intensity of the base color, resulting up to five different shades. The rest of the paper is organized as follows.

Section 2 describes architecture of the research methodology. Section 3 elaborates NC94 dataset and its storage technology. The ParaSQL queries employed in this work have been described in section 4 in sufficient detail. In section 5 we compile the related visualization concerns. Section 6 throws intense light on life in Iowa. Results have been discussed in section 7. Section 8 compiles the related work and the paper is concluded in section 9.

\section{ARCHITECTURE OF RESEARCH METHODLOGY}

The core concept of this work is to analyze the coldness and hotness in all counties of Iowa with the help of NC94 data available. Figure 1 is the complete architecture of the research methodology. A batch of commands is executed on NC94 climatic dataset via an integrated GUI based framework [13]. The commands are nothing but ParaSQL queries that run in a sequential order in a batch, starting from loading the data (if not already loaded) into CanStoreX to streaming the data out into a persistent text file. It is later read as input by a user defined java object, called Range. The function of Range object is to read the input text file and an XML file carrying the color coding scheme and calculate the number of cold days for every county in Iowa. The count on the cold/ hot days for a particular county reflects the degree of coldness/ hotness for that county. Subsequently (on-the-fly) it (Range) populates a java hash map by county's FIPS and associated degree of coldness as key-value pair and injects it to another user defined java object called Main. 


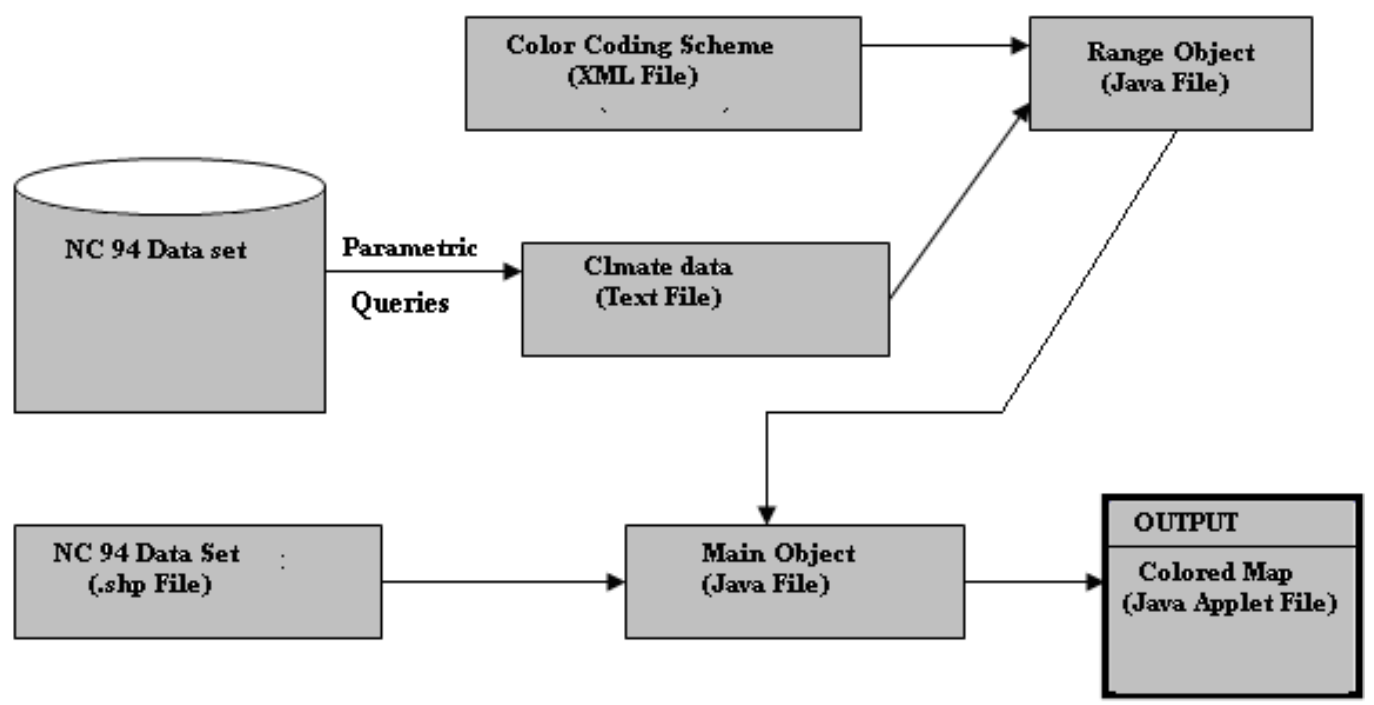

Figure 1. Architecture of research methodology

It can be seen in the figure a shape file of NC94 dataset is also used as an input to Main object. The shape file contains the various attributes for every county in all states of North Central Region. Out of which the counties in Iowa are of the interest for this work and rest of the states are ignored. The Main object reads all the counties (FIPS), but one at a time and it geographical shape from shape file and based on the degree of coldness/ hotness (injected from Range object) paints it with an appropriate shade of base color (blue for coldness and red for hotness).

\section{NC94 DATASET AND STORAGE TECHNOLOGY}

\subsection{NC94 Dataset}

The NC94 dataset is an agricultural dataset composed of climate, crop and soil data in North Central Region and collected by NCRA for the duration 1971-2000. It is used intensively to facilitate crop and risk analysis, pest management and forecasting. In addition, it is publicly accessed through the internet, allowing the public to ask ad-hoc queries [1]. In order to use the NC94 dataset for scientific purpose, many scientific data formats are used with software packages to store and process it for environmental development. Despite the advantages of the scientific data formats, it is not easy for the public to directly access the rich dataset stored in scientific data formats because they are not database management systems which directly support ad-hoc queries.

NC94 dataset contains the important spatial data of North Central Region of United States. Sharma and Gadia in [12] have used NC94 dataset and provided detailed description about it. In this work NC94 dataset is used in two formats; 1) shape file format, and 2) GML format. In [4] authors mention that IEM (Iowa Environmental Mesonet) has collected many important datasets of evolving atmospheric, soil and hydrologic fields for analysis and for dissemination and have provided the shape file format of NC94 dataset. We use the shape file format for the graphical display of all counties in Iowa whereas the GML format has been used to fetch the required attributes to calculate the degree of coldness in all 99 counties of Iowa. In [5] authors present a detail study on Geography Markup Language (GML), issues that arise from using GML for spatial databases and solutions that have been proposed. 


\subsection{Canonical Storage for XML (CanStoreX)}

CanStoreX is an in-house developed XML storage technology known as Canonical Storage for XML [2]. It ramifies an XML document into pages and stores them in the paginated form in the storage. The initial implementation of CanStoreX for storing XML document and DOM API, called DiskDOM for their access was undertaken by Shihe Ma [3], and used textual representation for pages, and a common Java based DOM API for navigation within pages. Thus every page is seen as a java based Node object by its DiskDOM. Due to heap-based management of memory for the objects in Java, the system would choke when paginating or accessing XML documents beyond 1 gigabyte range. Currently, binary pagination algorithm has been tested for XML documents up to 1 terabytes in size.

Pages within the storage are interconnected using special nodes called storage facilitating nodes $(s f n)$. Currently there are two types of $s f n$ available to facilitate: f-node, and c-node. The f-node is used to group one or more siblings having the same parent. A c-node contains a pointer to a child page where a subtree rooted at an f-node resides. Each page itself is organized as a self contained legal XML document. Thus the whole XML document becomes a tree of pages where each page, and XML document on its own right, is stored as a tree in a binary format. An XML document in CanStoreX remains in a ready state for access.

Previously only climate data was available and was stored in an older version of CanStoreX where XML was stored in text form. The newer binary version of CanStoreX allows a readily available tree-like navigation in the paginated XML document. Addition of crop and soil data requires different internal representation in order to achieve a uniform view for users that is at par with the climate data. Further, the internals were conformed to use the version of CanStoreX where pages are stored in binary, rather than text form.

\section{PARASQL QUERY}

Following batch of commands is used in this research work. The batch is responsible for loading the NC94 dataset to the storage, parsing the client query and creating a parse tree, building an expression tree, executing a query, and redirecting the resultant tuples to the output text file. All the commands are run through a integrated GUI framework, an in-house development. It provides a common platform to run the parametric query of different subsystems. To make GUI aware about a subsystem, a suffix is assigned before the command of that subsystem is issued e.g. NC94 :> loadNC94data climate where NC94 is the name of target subsystem. Once the data is uploaded (use LoadNC94Data) in local CanStoreX, it needs to be opened (use OpenNC94Database command) before any parametric query is run.

- LoadNC94Data -This command is used for invoking the loader module for loading the NC94 dataset into the common storage. Out of 'climate' or 'soil' or 'crop', this command takes any one of he relations as input parameter and loads the specific relation into the common storage.

- OpenNC94Database -This command opens the NC94 XML catalog and parses using DOM parser for accessing schema of the NC94 relations. It is an initialization step.

\section{- $\quad$ SELECT *}

RESTRICTED TO [[(C.MaxTemp + C.MinTemp)/2 $\leq 0]]$

FROM Climate C II queryop1.txt; 
This command is intended to collect the data suitable for coldness calculation. The fetched tuples are redirected to a text file, queryop 1.txt. It can be noticed here, only those tuples which have average temperature less than $0^{0} \mathrm{C}$ are stored in the file.

\section{- $\quad$ RELECT *
RESTRICTED TO [[(C.MaxTemp + C.MinTemp $) / 2 \geq 20]]$ FROM Climate C II queryop2.txt;}

This command is used to calculate the hotness and only those tuples which have average temperature greater than $20^{\circ} \mathrm{C}$ are stored into queryop 2 text file.

- CloseNC94Database -This command closes the handler to the NC94 relation in the storage and releases all the resources.

\section{VISUALIZATION CONCERNS}

\subsection{Color Coding Scheme}

In this section we show the color coding scheme. The figure 3 shows the $x m l$ representation of the contents needed for color coding. The tag Base is given the hexadecimal value (consisting of six digits) which corresponds to dark black color. In the Base tag, the left most two bits, middle two bits, and right most two bits are used to represent red color, green color and blue color respectively.

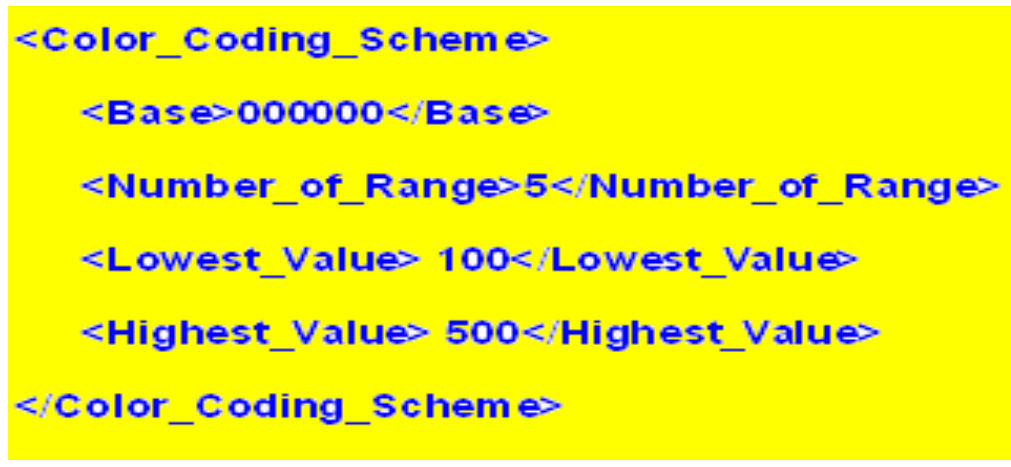

Figure 3. Color coding scheme

So in order to demonstrate the variable degree of coldness and hotness in different counties the right most two bits and left most two bits are manipulated respectively. Tag Number_of_Ranges shows the range of shades of a base color we use in the rendition of the map. The tag Lowest_Value and Highest_Value are the highest and lowest boundaries of degree of coldness/ hotness. These two limits are at substantial marginal difference from the actual value of the degree of coldness/ hotness in any county. 


\subsection{Algorithm to Calculate the Degree of Coldness and Hotness}

Following is the pseudocode to calculate the degree of coldness and hotness in a county. Line 1 helps to store the set of states extracted from NC94 dataset into an array. As for this research purpose we are interested to confine within Iowa only so the array in line 1 is iterated to extract the spatial data in Iowa only and variable $S$ in line 4 temporarily holds the state name. Line 5 helps to check whether the stored state in variable $\mathrm{S}$ is Iowa. Once line 5 returns true, all counties of Iowa are extracted and stored in another array CC as shown in line 7.

\section{ALGORITHM 1: To calculate the degree of coldness and hotness in a county}

1. Array SS $\leftarrow$ set of all states in North Central Region in NC94 dataset

2. WHILE (SS. length $>=0$ )

3. BEGIN

4. $S \leftarrow$ state extracted from array

5. IF ( $\mathrm{S}==$ ' 'Iowa')

6. BEGIN

7. Array CC $\leftarrow$ set of counties of Iowa

8. FOR ( int $\mathrm{a}=0 ; \mathrm{a}<\mathrm{CC}$.length; $\mathrm{a}++$ )

9.

10. BEGIN

11. Number of cold days: $\mathrm{n} 1 \leftarrow\left\{\left(\frac{\text { Temp.MAX }+ \text { Temp.MIN }}{2}\right) \leq 0\right\}$ Number of hot days: $\mathrm{n} 2 \leftarrow\left\{\left(\frac{\text { Temp.MAX }+ \text { Temp.MIN }}{2}\right) \geq 20\right\}$

13. Number of day in 30 years: $\mathrm{N}$

14. Degree of coldness $=\mathrm{n} 1 / \mathrm{N}$

15. Degree of hotness $=\mathrm{n} 2 / \mathrm{N}$

16. END

17. END

18. END

This array is iterated to calculate the number of days in which the average of maximum temperature and minimum temperature is less than 0 and greater than 20 and line 11 and 12 in this pseudocode correspond to those operations respectively. Once the numbers of cold days are calculated, then it is extremely straight forward to calculate the degree of coldness and hotness over a 30 years period and line 14and 15 in pseudocode help to return the degree of coldness and hotness of counties, one at a time, thus finally when the for loop in line 8 is exhausted, we obtain the calculated degree of coldness and hotness for all counties in Iowa.

\subsection{Model to Calculate the Darkness}

Let us assume that Highest_Value is the highest limit and Lowest_Value is the lowest limit of the degree of coldness/ hotness expected in any county. We supply these values in XML file. We further assume that Current_Value represents the actual degree of coldness/ hotness for a particular county calculated using Algorithm 1. Then the intensity of darkness of color is obtained as follows.

Intensity of darkness $=\frac{\text { Highest_Value }- \text { Current_Value }}{\text { Highest_Value }- \text { Lowest_Value }}$ 


\section{LIFE IN IOWA}

\subsection{Quality of life}

Iowa is an agricultural rich state and farming is considered as the primary source of income for Iowans [16]. Most of the families in Iowa live on farms and do physical hard work which keeps them healthy and help to live longer life [22]. There are other commercial states e.g. California in USA which have numerous industries as the source of income and attract a large pool of immigrants from various parts of the world from employment perspective. This infuses huge competition for employment which needs a fast pace life to cope up with that leads towards tense life which intern reduces the life expectancy [22]. Unlike these states majority of population in Iowa depends on agriculture and posses permanent resources for their earning and living. It does not attract huge immigrants from outside resulting less competition. So Iowans do the work at per their pace and enjoy less stressful lifestyle which plays a major role in increasing life expectancy [22]. The living expanses in Iowa are very cheap [17]. So due to the calm and easy lifestyle and lower costs of living, Iowans do not prefer to migrate to other states.

Iowa is the second state among the 50 states in United States which have total state population (in percentage) that are centenarians [15]. In the centenarian - related records maintained by IDA, there are $85 \%$ women of total population and majority of them reside in facilities and but around $1 / 4$ still live in their own home or with relatives. As per the records there is one super centenarian in Iowa: name - Neva Morris, age - 114 years, place - Ames.

\subsection{Climate Impact in Iowa}

There are various climatic factors which impact human life. In this paper, the temperature is considered as the prime factor that potentially impacts human life especially senior citizens.

- Temperature - The average temperature over the year is between $45^{\circ} \mathrm{F}$ in the extreme north to 52 in the southeastern corner of the State. July is the hottest month, with daily temperatures ranging from 61 to $82^{\circ} \mathrm{F}$ in the northeast corner of the State. January is considered as the coldest month where temperature ranges from 4 and $22^{\circ}$. The interested reader can read the document written in [18].

Overall, Iowa's climate is nearly suitable and ideal to the grain crops such as corn and soybeans production. In this paper the climate concerns are studied exclusively from senior citizen's prospective with the objective to access the counties with lowest climate variations over the year with the belief that nearly uniform climate exposure will help in comfortable life.

\subsection{Smart Home Involvement}

Due to older age a senior resident may encounter with different impairments: motor, physical, social, cognitive, and dementia etc. Few of them may be lighter and healable but some of them are too severe to cure. In these unfavorable conditions an old person needs a caregiver all the time in order to avoid any unwanted situation. In today's competitive world due to busy schedule it is too difficult for a caregiver to be available with elderly all the time. To combat these difficult situations, the concept of smart home has come into existence and serves as a help not only to elderly but also to caregiver as well. It absorbs lot of responsibilities of a caregiver. Smart homes are intelligent, technical, and automated homes which are employed to help elderly. Thus smart 
home is an assistive tool to the caregivers and senior resident both. To a caregivers, it assists her/ him to make sure their alone living parent is alive, living well, and performing all the tasks necessary for their well-being every day [19] and for a senior resident it elevates her/ his life by providing independency. Finding a suitable place to establish smart home is a great concern and the results of this paper may help by finding out the counties with least adverse change in climate where the development of smart home will help efficiently to improve the life of senior citizens in Iowa.

\section{RESULTS}

In this section the results of this research work have been populated. The coldness and hotness have been plotted separately into two different maps. Figure 4 shows the degree of coldness in all 99 counties of Iowa. There are five different shades of blue color have been used and each and every county is painted with a shade of blue color. The shade with higher intensity reflects the higher degree of coldness and the shade with lower intensity reflects the lower degree of coldness in a particular county for duration of 30 years. In NC94 dataset precipitation is the base for coldness calculation.

Figure 5 is a map showing the degree of hotness in all 99 counties of Iowa. Similar to the color coding scheme used in coldness calculation, there are five different shades have been used to plot the hotness and every county is painted with a shade of reddish/ yellow color. The yellow color reflects the least hot county and the firebrick reflects the hottest county in Iowa over the 30 year duration and can be considered as extreme temperatures. The counties painted with reddish shades have temperature in between these two temperatures.

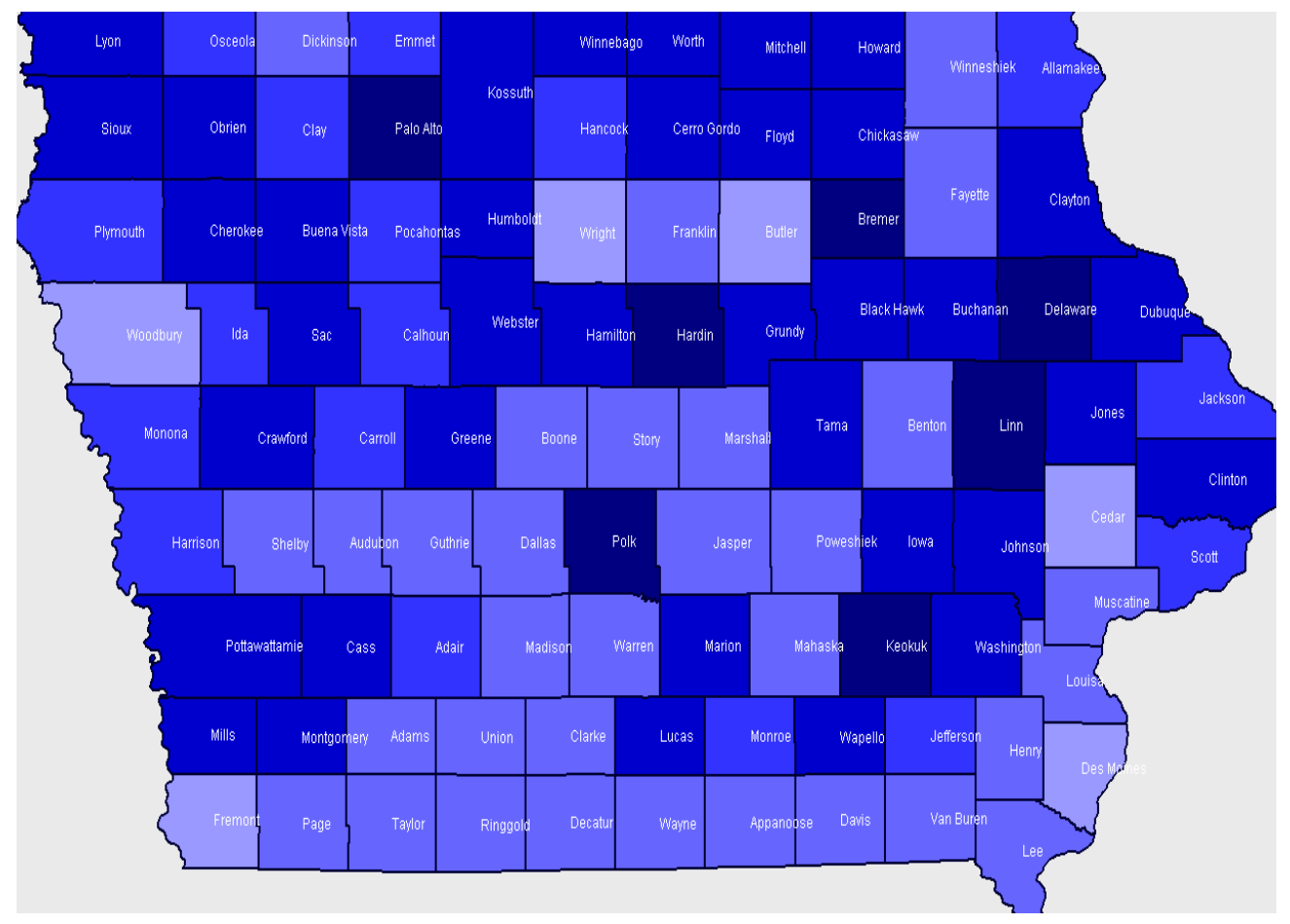

Figure 4. The display of degree of coldness in the counties of Iowa 


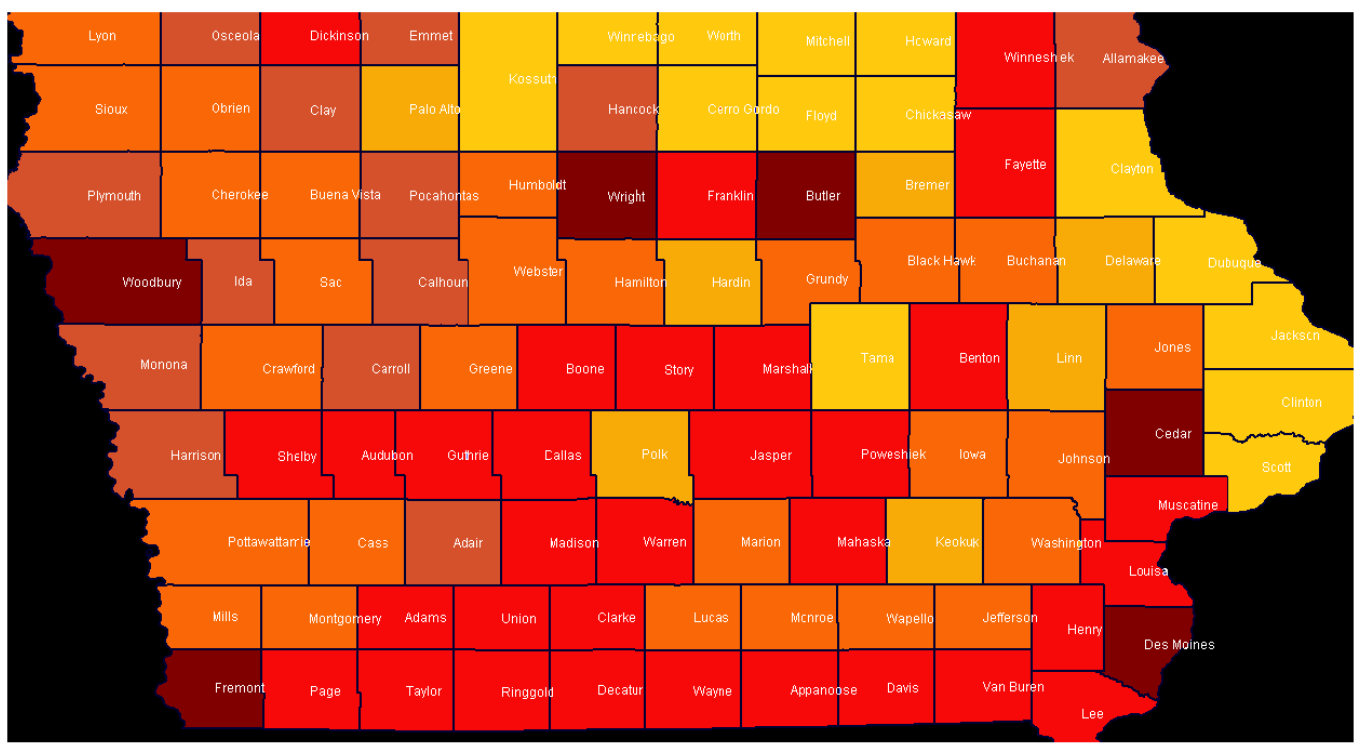

Figure 5. The display of degree of hotness in the counties of Iowa

\subsection{Climatic Analysis}

In this research work, the analysis of the results carries great potential and careful analysis is utmost important. The objective is to find out those counties which have least temperature variation over the 30 years period. By analyzing the figure 4 and figure 5 it can be observed that most of the counties at up north of Iowa are colder and the counties in the down south are hotter. The majority of counties at the west central Iowa e.g. Plymouth, Ida, Monona, Harrison and many others with the same shade of color, can be considered as the moderate suitable counties because of the least variation in the temperature. This geographical climate analysis may be useful in several other studies for example; a primary consideration can be given to choose more suitable sites in order to establish the smart homes for senior citizens.

\section{RELATED WORK}

In [6] author has compiled the detailed discussion about the common GUI and its command execution. He has discussed each and every features of GUI and talked about all parametric commands useful to process NC94 dataset. In paper [7] authors have given a broad description of XML language with additional features. Authors in paper [8] give a tour of a database management system designed from scratch for storing and processing XML data, called Natix. This motivated Ma [3] for the development of CanStoreX technology. The paper [9] describes the overall design and architecture of the Timber XML database system, based upon bulk algebra for manipulating trees, and natively stores XML. This also worked as a strong motivation behind the development of CanStoreX technology. In the paper [10] authors present an XML-based implementation methodology for the parametric temporal data model and in the implementation, they develop a new XML storage called CanStoreX (Canonical Storage for XML) and build the temporal database system on top of the CanStoreX. In [11] North Central Regional Association of State Agricultural Experiment Station Directors (NCRA) develops a strategy for data collection that helps in study, efficient crop management and to reduce the risk factors associated with agriculture practices, which depends on various factors. The collection and organization of data based on the states in the north-central region of the United States is mentioned as the primary goal of the Association and climate, crop and soil data is collected for these states. The result of data collection and organization efforts is the NC94 dataset which we use extensively in 
our research work. In [12] authors briefly describe the attempt to calculate the degree of coldness in Iowa and in [13] that authors liberally explore the calculation and analysis of coldness in Iowa. It is well known fact that XML is powerful to provide configuration. In [14] the potential of XML has been explored one again at configuration level. By varying the values of range in XML configuration file, the shades of color on map can vary distinctly which is useful to the people with defective eyesight. Sharma et al. use Perl Status Reporter, SRr [20] as an attempt for spatiotemporal data mining [21].

\section{CONCLUSION AND FUTURE WORK}

We have calculated and analyzed the cumulative degree of coldness and hotness in all counties of Iowa over 30 years (1971-2000) duration. The degree of coldness has been depicted using blue as the base color whereas for the hotness, red color is used. The increase and decrease in degree of coldness/ hotness is analogous to the higher and lower intensity of the base color respectively. After the analysis it can easily be accessed that there are few counties in Iowa have least climate variation in terms of coldness and hotness and are good to stay for senior citizens and the concept of smart home would help more effectively. We expect that the obtained results will provide benefits to senior citizens and agricultural community and may be useful for XML, GIS, database, Iowa Department on Aging, and other scientific community in future research.

The future task involves the calculation of other climate related factors and to analyze them more closely from agriculture and human life perspectives.

\section{REFERENCES}

[1] Seo-Young N.: Hybrid Storage Design for NC94 Database within the Parametric Data Model Framework. In: Proceedings of the International Conference on Computational Science and Its Applications, Part II, pp. 145-154, Glasgow, UK, May 8-11, 2006.

[2] Patanroi, D.: Binary Page Implementation of a Canonical Native Storage for XML.Master's thesis, Department of Computer Science, Iowa State University, Ames, Iowa (August) 2005.

[3] Ma, S.: Implementation of a canonical native storage for XML. Master's Thesis, Department of Computer Science, Iowa State University, 2004.

[4] Gadia S.K., Gutowski W. J., Al-Kaisi M., Taylor S.E., and Herzmann, D.: Database tools promoting extensive, user-friendly access to the iowa environmental mesonet. Baker Proposal (2004).

[5] Lakshmi N. Sripada, Chang-Tien Lu, and Weili Wu.: Evaluating GML Support for Spatial Databases. In: 28th Annual International Computer Software and Applications Conference - Workshops and Fast Abstracts - (COMPSAC'04) compsac, vol. 2, pp.74-77, 2004.

[6] Narayanan, V.: A Workbench for Advanced Database Implementation and Benchmarking. Master's thesis, Department of Computer Science, Iowa State University, Ames, Iowa, 2009.

[7] Bray T., Paoli J., Sperberg-McQueen C.M. , Maler E., and Yergeau F.: Extensible- Markup Language (XML) 1.0. (Fourth Edition) W3C recommendation, 16 August 2006.

[8] Fiebig T., Helmer S., Kanne C. -C., Moerkotte G., Neumann J., Schiele R., and Westmann,T.: Anatomy of a native XML base management system. Springer-Verlag, 2002.

[9] Jagadish H.V., Al-Khalifa S., Chapman A., Lakshmanan L.V.S., Nierman A., Paparizos S., Patel J.M., Srivastava D., Wiwatwattana N., Wu Y., and Yu C.: TIMBER: A native XML database. VLDB Journal, 11(4): 274-291, 2002. 
[10] Noh S.-Y., Gadia, S. K., and Ma, S.: An XML-based methodology for parametric temporal database model implementation. Journal of Systems and Software, Volume 81, Issue 6, June 2008.

[11] North Central Regional Association of State Agricultural Experiment Station Directors. Expected Outcomes. NC094: Impact of Climate and Soils on Crop Selection and Management. September2004.

[12] Sharma S., and Gadia S. K., "On the Calculation of Coldness in Iowa, a North Central Region, United States: A Summary on XML Based Scheme in Spatial Database (NC94),"International Conference on Advances in Information and Communication Technologies (ICT 2010), Springer CCIS series.

[13] Sharma S., and Gadia S. K., "On Analyzing the Degree of Coldness in Iowa, a North Central Region, United States: An XML Exploitation in Spatial Database (NC94)," First International Workshop on Database Management Systems (DMS 2010), Springer CCIS series , July 23-25, 2010.

[14] Sharma S., and Gadia S. K., "An XML-Based Range Variation Approach to Render the Coldness in Iowa, a North Central Region, United States," IEEE - Second International Conference on Information Management and Engineering (ICIME 2010), April 2010.

[15] Iowa Department on Aging. [Online] http://www.aging.iowa.gov/resources/agingstats.html.

[16] United States Department of Agriculture, National Agriculture Statistics Service. [Online] http://www.nass.usda.gov/QuickStats/PullData_US.jsp,

http://usda.mannlib.cornell.edu/usda/current/AgriPricSu/AgriPricSu-08-05-2009.pdf, http://www.nass.usda.gov/Charts_and_Maps/Farm_Labor/fl_allwg.asp.

[17] Iowa life changing. [Online] http://www.iowalifechanging.com/business/qualityoflife.aspx

[18] Climate in Iowa. [Online] http://www.crh.noaa.gov/images/dvn/downloads/Clim_IA_01.pdf

[19] Smart Houses Keep Eye on Elderly. [Online] http://marc.med.virginia.edu/library_techtv.html

[20] Sharma S., Cohly H., and Pei T., "On Generation of Firewall Log Status Reporter (SRr) Using Perl," International Journal of Network Security \& Its Applications, Vol. 1 No. 2, pp. 90-99, July 2009.

[21] Sharma S., and Gadia S., "Perl Status Reporter (SRr) on Spatiotemporal Data Mining," Inter-national Journal Computer Science and Engineering Survey," (AIRCC- IJCSES), August, 2010.

[22] Stibich M., "Simple Steps to Increase Your Life Expectancy." [Online] http://longevity.about.com/od/liveto100/ss/life-expectancy_7.htm

\section{Authors}

Shashi Gadia obtained his $\mathrm{PhD}$ in Mathematics from University of Illinois, Urbana in 1977. Presently he is an associate chair in Computer Science Department at Iowa State University. His main research interest is in concept of non-atomic values in databases - values with dimensions such as time, space, and beliefs on one hand and hierarchical values such as those in XML on the other. He has worked in temporal, spatial, and multilevel security databases, optimization, incomplete information, query languages. He pioneered temporal databases.

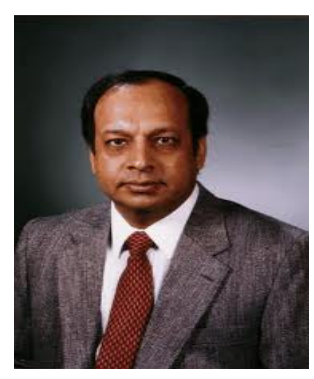


Sugam Sharma is currently perusing PhD in Computer Science at Iowa State, USA. He works in interdisciplinary research domains with following research interests: Databases-Spatial databases: Climatic data, GIS and XML, Wireless Sensor Network, Smart Home Technology, and Bioinformatics. He has worked as an asst. professor, a researcher at Trent Lott Center for Geospatial Research, USA, and a software developer (J2EE) in NATVTEQ-Traffic.com, PA, USA. He is editor in-chief (executive), for IJCIB.

Niranjan Kumar received his MS in Computer Science from Iowa State University in 2008 where his advisor was Dr.Shashi Gadia. His research involved porting the NC-94 dataset to the CanStoreX platform and modifying the existing query interface to work with this paginated data. $\mathrm{He}$ is currently working as a software engineer for Cerner Corporation, Kansas City, Missouri.

Valliappan Narayanan obtained his Bachelor's in Information Technology from India in the year 2006 and worked for one year in Honeywell, India as a Software Engineer before coming for Master's. $\mathrm{He}$ completed his Master's in Computer Science from Iowa State University in the year 2009 under the Guidance of Dr.Shashi K. Gadia in Databases and XML. Currently, He is working as a Software Engineer in Intel, Oregon, USA.

Xinyuan Zhao is currently working toward the $\mathrm{PhD}$ degree in Computer Science Department at Iowa State University. His research interests include XML databases, XQuery language, and database implementation.
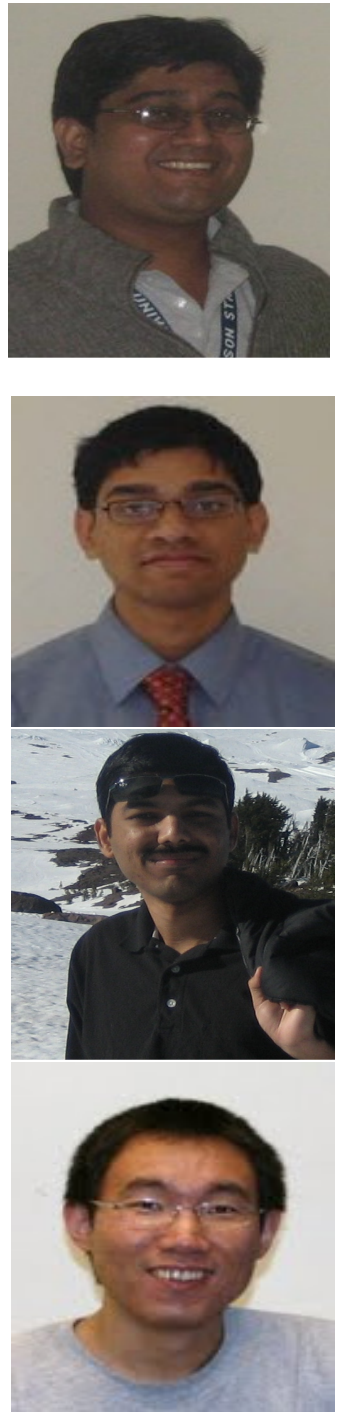